\title{
La biodiversité spécifique des ressources démersales du plateau continental guinéen : utilisation d'indices classiques pour un diagnostic sur l'évolution de l'écosystème
}

\author{
Jérémy Lobry ${ }^{\mathrm{a}, *, 1}$, Didier Gascuel ${ }^{\mathrm{a}}$, François Domain ${ }^{\mathrm{b}}$ \\ ${ }^{a}$ Ensar halieutique, 65, rue de Saint-Brieuc, 35042 Rennes cedex, France \\ ${ }^{b}$ IRD-CNSHB, BP 1984 Conakry, Guinée
}

Reçu le 14 octobre 2002 ; accepté le 3 février 2003

\begin{abstract}
Résumé
La diversité spécifique est généralement considérée comme un facteur-clé de la résilience des écosystèmes en réponse à la pression anthropique et notamment à la pression de pêche. Dans cet esprit, on analyse ici l'impact de la pêche sur la diversité spécifique, à partir du cas des ressources démersales côtières de Guinée. Celles-ci ont la particularité d'avoir subi récemment un très rapide et important accroissement de la pression de pêche. En une quinzaine d'années, on est ainsi passé d'une situation dans laquelle les ressources démersales étaient considérées comme étant " à l'état vierge », à une surexploitation globale. Cette évolution mérite d'être considérée comme un « modèle accéléré » de l'histoire d'une exploitation. L'analyse se fonde sur les données de 18 campagnes scientifiques de chalutage démersal effectuées dans la zone côtière guinéenne entre 1985 et 1992. Le niveau de diversité spécifique, par campagne ou par trait de chalut, est quantifié par des indices synthétiques : la richesse spécifique, la diversité de Shannon et l'équitabilité. Les indices par trait de chalut sont analysés par les méthodes de modélisation en analyse de variance, afin de dégager une évolution temporelle des indicateurs. Les diagrammes rang/fréquence de chaque campagne sont également établis. On montre que les indices de diversité et d'équitabilité restent sensiblement constants, alors que l'abondance et la richesse spécifique diminuent significativement au cours de la période. Les espèces les moins abondantes et particulièrement des espèces de la communauté à Sparidés, tendent ainsi à disparaître des prélèvements. Enfin, l'année 1989 présente des indices de diversité faibles, liés à des conditions environnementales favorables pour certaines espèces entraînant un recrutement exceptionnel de celles-ci. La pertinence des indices utilisés est discutée.
\end{abstract}

(C) 2003 Éditions scientifiques et médicales Elsevier SAS and Ifremer/IRD/Inra/Cemagref. Tous droits réservés.

\begin{abstract}
Fish biodiversity of the Guinean demersal resources. Use of classical indices for a diagnosis on the evolution of the ecosystem. Species diversity is usually regarded as a key factor of ecosystem resiliency facing human impact and especially fishing pressure. This study analyzed the impact of fishing on fish species diversity. We focused on the Guinean coastal demersal resources which have recently undergoes very fast and significant increase in fishing pressure. Within a period of 15 years, the demersal resources evolved from a "virgin status" to a globally overexploited situation. This quick evolution can be regarded as a "model" of the history of an exploitation. The analysis is based on the data of 18 demersal trawling scientific campaigns carried out in the coastal zone between 1985 and 1992 . The level of the species diversity was quantified for each campaign and by trawl using different indices: species richness, the Shannon and equitability indices. The indices by trawl were analyzed by ANOVA methods in order to release a temporal evolution of the indicators. The rank/frequencies diagrams of each campaign were also drawn. The Shannon and equitability indices remained appreciably constant, whereas abundance and species richness decreased significantly during the period. The least abundant species, and particularly species from the Sparidae community, tended to disappear from the samples. Lastly, all diversity indices were weak in 1989. This can be related to suitable environmental conditions for the recruitment of some species. Relevance of the indices used is discussed.
\end{abstract}

(C) 2003 Éditions scientifiques et médicales Elsevier SAS and Ifremer/IRD/Inra/Cemagref. All rights reserved.

Keywords: Biodiversity; Species diversity; Shannon index; Demersal resources; Guinea

* Auteur correspondant.

Adresse e-mail : jeremy.lobry @bordeaux.cemagref.fr (J. Lobry).

${ }^{1}$ Adresse actuelle : Cemagref, Ressources aquatiques continentales, 50, avenue de Verdun, 33612 Cestas cedex, France (C) 2003 Éditions scientifiques et médicales Elsevier SAS and Ifremer/IRD/Inra/Cemagref. Tous droits réservés.

DOI: 10.1016/S0990-7440(03)00010-X 


\section{Introduction}

L'étude des impacts induits par la pêche sur les ressources marines exploitées s'est jusqu'à présent essentiellement construite avec des approches mono-spécifiques conduisant à la recherche d'une utilisation raisonnée des différents stocks exploités. Ce n'est que récemment que des préoccupations de nature plus écosystémique se sont fait jour (May, 1976 ; Koslow et al., 1988 ; Jennings et Polunin, 1996 ; Shin, 2000). Elles conduisent à s'interroger sur les modifications qu'entraînent les pressions anthropiques et particulièrement halieutiques, sur la structuration et le fonctionnement des écosystèmes et leurs capacités de réaction. À ce titre, la biodiversité spécifique des écosystèmes marins exploités est généralement considérée comme un facteur clé de leur résilience (Koslow et al., 1988 ; Lévêque, 1994 ; Naeem et Li, 1997 ; Russ et Alcala, 1998).

La présente étude s'inscrit dans cette thématique scientifique extrêmement large (approche écosystémique des milieux marins et de leur exploitation). Elle se focalise sur une question particulière qui est celle de la quantification de la biodiversité spécifique et de sa variabilité, en relation avec la pression anthropique de pêche. De nombreux travaux ont déjà été menés dans cette direction, mais ils ont généralement pris en compte des écosystèmes marqués par une exploitation intense depuis de nombreuses années, voire depuis de nombreuses décennies (par exemple Blanchard, 2001). Faute d'une modification importante de l'intensité d'exploitation, aucun changement notable de la biodiversité ne semble pouvoir être clairement mis en évidence. Dans cette optique, l'exploitation des ressources côtières de Guinée constitue un cadre d'étude particulièrement intéressant.

En effet, comme dans nombre de pays africains mais sans doute plus qu' ailleurs, l'exploitation halieutique des ressources marines s'est développée de manière extrêmement rapide en Guinée, au cours des 2 dernières décennies (Gascuel et al., 1997 ; Domain et al., 2000). Ceci est particulièrement vrai pour les ressources démersales côtières : jusqu' au milieu des années 1980, elles étaient dans une situation généralement considérée comme vierge (Domain, 1989); en une quinzaine d'années, elles ont atteint une situation de surexploitation (Gascuel et al., 1997). Cette évolution mérite d'être considérée comme un «modèle accéléré » de l'histoire d'une exploitation. Dans un contexte comme celui-ci, on peut imaginer que de profondes modifications du fonctionnement de base de l'écosystème et de sa structure même ont pu survenir.

L'objectif de ce travail est d'analyser l'évolution de la biodiversité spécifique des ressources démersales de Guinée au moyen d'indices classiques issus essentiellement de l'écologie théorique. Ces indicateurs simples sont le plus souvent utilisés en écologie terrestre et en phytosociologie (Frontier, 1983 ; Barbault, 1992). On s'attachera ainsi dans le même temps à mesurer à quel point ils peuvent être utiles et pertinents pour diagnostiquer l'évolution d'un écosystème marin dans un contexte de très rapide accroissement de la pression de pêche. Cette étude s'appuie sur l'analyse de données issues des campagnes scientifiques de chalutages de fond menées sur la partie côtière du plateau continental entre 1985 et 1992.

\section{Matériel et méthodes}

\subsection{Cadre de l'étude}

La Guinée Fig. 1) possède le plus vaste plateau continental de l'Afrique de l'Ouest, avec en particulier des fonds inférieurs à $20 \mathrm{~m}$, généralement vaseux, qui s'étendent jusqu'à $90 \mathrm{~km}$ au large au nord. Au niveau hydroclimatique, le climat local oscille entre une saison sèche et froide de novembre à avril (caractérisée par la présence d'un upwelling au nord-ouest) et une saison humide et chaude de mai à octobre (caractérisée par de fortes précipitations).

Le peuplement ichtyologique côtier est essentiellement représenté par 2 communautés écologiques sensiblement différentes (Fager et Longhurst, 1968 ; Domain, 1989) (Tableau 1):

- la communauté à Sciaenidés a une répartition très côtière. Elle fréquente les eaux chaudes et dessalées jusqu'à une quinzaine de mètres de profondeur. De fortes biomasses sont associées à l'abondance locale de vases, qui constituent des fonds écologiquement riches ;

- la communauté à Sparidés a une aire de répartition située plus au large, sur des fonds de 20 à $60 \mathrm{~m}$. Les espèces de cette communauté fréquentent les eaux plus froides et salées. Les densités sont faibles.

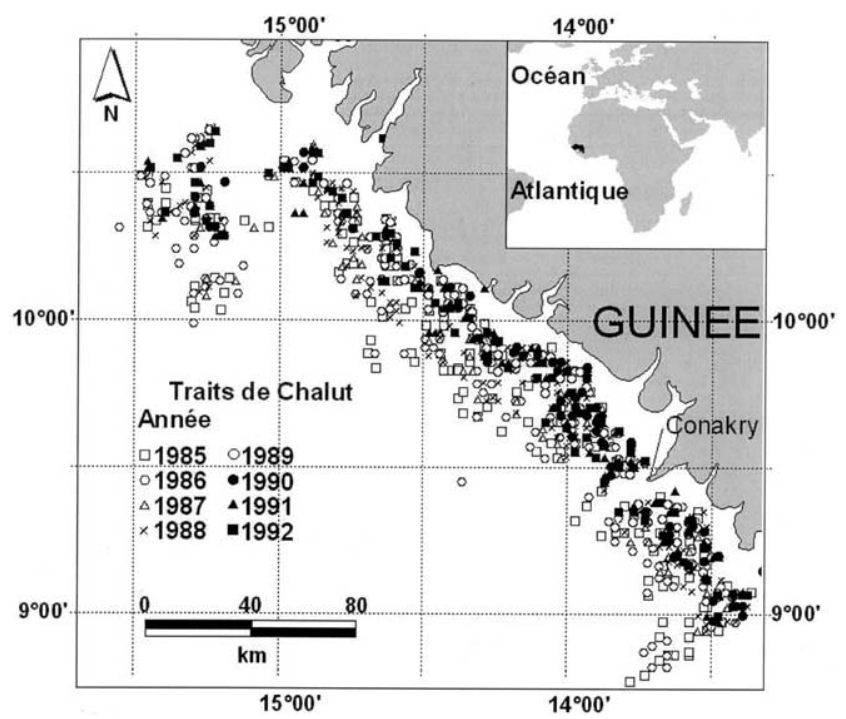

Fig. 1. Zone littorale et plateau continental guinéen. Localisation des traits de chalut réalisés (seuls, les traits situés entre la côte et la ligne pointillée sont pris en compte dans l'analyse ; cf. texte).

Fig. 1. The Guinean coastal zone. Location of the trawl (only trawl between the coast and the dotted line are taken into account in the analysis. See text). 
Tableau 1

Liste des espèces caractéristiques des 2 principales communautés écologiques présentes sur le plateau continental guinéen (d'après Domain, 1989)

Table 1

Fish species of the 2 main ecological communities of the Guinean coastal zone (Domain, 1989)

\begin{tabular}{|c|c|c|}
\hline \multicolumn{3}{|l|}{ La communauté à Sciaenidés } \\
\hline Albula vulpes & Dasyatis hastata & Pomadasys jubelini \\
\hline Arius heudelotii & Dasyatis rutis & Pomadasys rogerii \\
\hline Arius latiscutatus & Dicologlossa hexophtalma & Pseudotolithus brachygnatus \\
\hline Arius parkii & Drepane africana & Pseudotolithus elongates \\
\hline Brachydeuterus auritus & Elops lacerta & Pseudotolithus epipercus \\
\hline Chaetodipterus goreensis & Galeoides decadactylus & Pseudotolithus hostia moori \\
\hline Chaetodipterus lippei & Gymnura haltavela & Pseudotolithus senegalensis \\
\hline Chloroscombrus chrysurus & Gymnura micrura & Pseudotolithus typus \\
\hline Cynoglossus cadenati & Ilisha africana & Sphyraena barracuda \\
\hline Cynoglossus canariensis & Mugil sp. & Sphyraena guachancho \\
\hline Cynoglossus monodi & Pentanemus quinquarius & Sphyraena sphyraena \\
\hline Cynoglossus senegalensis & Polydactylus quadrifilis & Trichiurus lepturus \\
\hline Dasyatis margarita & Pomadasys incisus & \\
\hline \multicolumn{3}{|l|}{ La communauté à Sparidés } \\
\hline Dactylopterus volitans & Epinephelus alexandrinus & Priacanthus arenatus \\
\hline Dentex canariensis & Ephippion guttifer & Psettodes belcheri \\
\hline Dentex filosus & Lagocephalus laevigatus & Pseudupeneus prayensis \\
\hline Epinephelus aeneus & Pagellus bellottii & Sparus caeruleostictus \\
\hline
\end{tabular}

Deux types de pêches coexistent en Guinée. La pêche artisanale est pratiquée à partir de pirogues, parfois motorisées (le taux de motorisation est inférieur à $50 \%$ ), en utilisant des engins très divers. Elle représente $69 \%$ des débarquements en 1995 (52 $000 \mathrm{t}$, dont $23000 \mathrm{t}$ de poissons démersaux, pour 2300 pirogues) ; elle vise essentiellement les ressources très côtières de la communauté à Sciaenidés. La pêche industrielle (le complément : 23300 t pour 117 licences) est pratiquée au chalut, en principe dans les zones plus au large, mais elle vise également les ressources côtières (Chavance et al., 1997). Entre 1985 et 1991, on a assisté à un accroissement rapide de la pression de pêche en Guinée (Gascuel et al., 1997 ; Domain et al., 2000). Ce phénomène est mis en relation avec une diminution des captures et notamment des captures lors des campagnes d'échantillonnages Fig. 2.

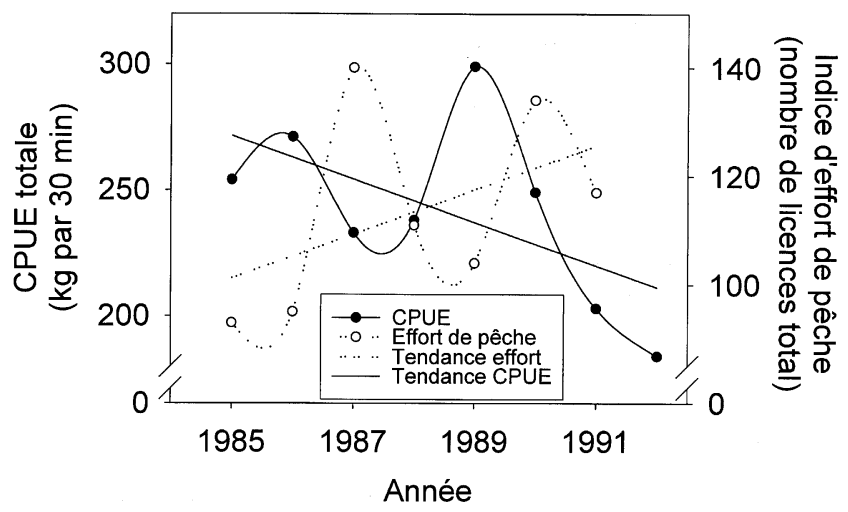

Fig. 2. Évolution comparée des CPUE lors des campagnes d'échantillonnages (en $\mathrm{kg} / 30 \mathrm{~min}$ ) et de l'effort de pêche (en nombre de licences total pour la pêche industrielle).

Fig. 2. Evolution of CPUE during sampling campaign $(\mathrm{kg} / 30 \mathrm{~min})$ and fishing effort (total number of licences for industrial fisheries).

\subsection{Données}

On analyse ici les données issues de 18 campagnes de chalutage démersal réalisées de mars 1985 à avril 1992 par le N/O «A. Nizery » (campagnes Chagui, réalisées par l'Institut de Recherche pour le Développement, IRD et le Centre National des Sciences Halieutiques de Boussoura - CNSHB). Deux campagnes sont en principe réalisées par an : l'une en saison sèche et l'autre en saison humide. Différents impondérables (carénage ou panne du navire, problèmes de financement...) perturbent cependant fortement ce calendrier théorique tant au niveau du nombre de traits réalisés qu'au niveau des dates de campagne Tableau 2.

La zone échantillonnée couvre la partie la plus côtière du plateau continental guinéen. On note cependant, entre les premières et les dernières campagnes, une nette rétraction vers la côte de la zone échantillonnée résultant d'un choix stratégique du centre de recherche de recentrer les travaux sur la zone côtière Fig. 1. Afin de s'affranchir de ce changement de plan d'échantillonnage, seuls les traits de chalut effectués dans la partie la plus littorale sont pris en compte dans la présente analyse (1031 traits).

L'ensemble de ces traits est réalisé selon un protocole standard ; en particulier, la surface échantillonnée est considérée constante. Chaque trait de chalut est caractérisé par ses coordonnées (longitude et latitude), sa date, ses caractéristiques hydrologiques (température et salinité) et par le nombre d'individus et la biomasse de chaque espèce prélevée. Nous avons choisi ici de travailler avec des abondances exprimées en effectifs, ce qui semble mieux cadrer avec l'étude de la répartition spatio-temporelle des ressources et permet une meilleure adéquation avec l'approche écologique du problème (Jouffre et Domain, 2000). 
Tableau 2

Calendrier des campagnes CHAGUI (d'après Gascuel et al., 1997). Le nombre de traits réalisés est noté entre parenthèses à côté du numéro de la campagne Table 2

Calendar of CHAGUI campaigns (from Gascuel et al., 1997). Number of trawls is noticed into brackets

\begin{tabular}{|c|c|c|c|c|c|c|c|c|c|c|c|c|}
\hline & Janv. & Fév. & Mars & Av. & Mai & Juin & Juill. & Août & Sept. & Oct. & Nov. & Déc. \\
\hline 1985 & & & $1(81)$ & & & & & & & $2(80)$ & & \\
\hline 1986 & & & $3(79)$ & & & & & & & $4(80)$ & & \\
\hline 1987 & & & & & & & & & & & $5(79)$ & \\
\hline 1988 & & & & $6(88)$ & & & & & $7(90)$ & & & \\
\hline 1989 & & & & & & & & & $8(48)$ & & $9(46)$ & \\
\hline 1990 & & & & $10(25)$ & & & $11(32)$ & & & & & \\
\hline 1991 & $12(65)$ & & $13(80)$ & & & $14(78)$ & & & $15(80)$ & & $16(69)$ & \\
\hline 1992 & $17(80)$ & & & $18(80)$ & & & & & & & & \\
\hline Saison & Sèche & & & & & Humide & & & & & Sèche & \\
\hline
\end{tabular}

\subsection{Calcul des indices de diversité}

Trois types d'indices, classiques en écologie, sont calculés :

- la richesse spécifique $\mathrm{S}$ correspond au simple comptage du nombre d'espèces présentes dans l'échantillon ;

- l'indice de diversité de Shannon H' (Shannon, 1948) est utilisé en écologie comme mesure de la diversité spécifique (Margalef, 1958 in Frontier, 1983) :

$$
\mathrm{H}^{\prime}=-\sum_{i=1}^{S} \frac{n_{i}}{N} \times \log _{2} \frac{n_{i}}{N}
$$

avec : $S$ le nombre total d'espèces présentes ; $n_{i}$ l'effectif de l'espèce i dans l'échantillon et $N$ l'effectif total. $\mathrm{H}^{\prime}$ varie entre 0 , dans le cas où le peuplement n'est constitué que d'une seule espèce et $\log _{2} \mathrm{~S}$ dans le cas où toutes les espèces présentes le sont avec une abondance équivalente (généralement $\mathrm{H}^{\prime}=4,5$ ou 5 pour les peuplements les plus diversifiés). Dans la suite, le terme de « diversité » est employé en référence à l'indice de Shannon ;

- l'indice d'équitabilité R (Pielou, 1966) permet de mesurer l'équitabilté (terme que certains écologues réfutent, lui préférant celui de régularité) ou encore l'équirépartition des espèces du peuplement par rapport à une répartition théorique égale pour l'ensemble des espèces (Barbault, 1992) :

$$
\mathrm{R}=\frac{H^{\prime}}{H_{\max }} \text { avec }: H_{\max }=\log _{2} S
$$

La valeur de $R$ varie entre 0 (une seule espèce domine) et 1 (toutes les espèces ont la même abondance). L'équitabilité prend en compte la diversité potentielle maximale du système $\left(\mathrm{H}_{\text {max }}^{\prime}\right)$; c'est-à-dire la capacité du système à accepter $\mathrm{S}$ espèces en proportions équivalentes. $\mathrm{R}$ constitue donc une sorte de synthèse des 2 autres indices.

Ces différents indices sont calculés de 2 manières : par trait de chalut et par campagne. Dans le premier cas, on mesure ainsi une diversité locale du peuplement. Ces mesures par trait font ensuite l'objet d'une analyse statistique afin de déterminer les principaux facteurs influençant la diversité du peuplement local. À l'issue des calculs par campagne, on rapporte le plus souvent le résultat à l' année en effectuant une moyenne des valeurs obtenues pour les campagnes d'une même année.

Cette analyse est complétée par l'étude des diagrammes rang/fréquence, afin de voir l'évolution conjointe de la diversité et de la régularité de l'écosystème entre 1985 et 1992.

\subsection{Analyse statistique des données par trait, modèles d'analyse de variance}

Souvent utilisés pour des études spatio-temporelles, les modèles linéaires d'analyse de variance (LM : Linear Model) ont ici vocation à mettre en évidence la distribution spatiale (sans préjuger une éventuelle corrélation spatiale) des indicateurs écologiques et leur évolution dans le temps. Par une analyse de variance à plusieurs facteurs, on recherche ainsi une répartition spatiale et saisonnière moyenne (i.e. commune à l'ensemble des campagnes) et une variabilité distinguant les différentes campagnes ou les différentes années (Gascuel et al., 1997). L'analyse prend donc en compte des facteurs temporels (effet an et effet saison), spatiaux (effets bathymétrie et zone) et éventuellement spatio-temporels (effets croisés de type an*bathymétrie et an*zone). L'analyse de ces effets croisés est particulièrement intéressante car elle permet notamment de décrire l'évolution temporelle d'une variable par strate bathymétrique ou par zone géographique. Les modèles utilisés sont de type additif. En effet, la distribution des variables étudiées s'approche plus de celle de la loi normale que de la loi log-normale et aucun a priori particulier ne justifie une transformation logarithmique préalable. Il est donc apparu que l'emploi du modèle additif était le plus simple. On retient un modèle avec les facteurs significatifs parmi les suivants : an, saison, bathymétrie, zone, an*saison, an*bathymétrie, an*zone, zone*bathymétrie voire an*bathymétrie*zone Tableau 3. On teste aussi les effets éventuels des facteurs de l'environnement tels que la 
Tableau 3

Définition des variables utilisées pour la modélisation linéaire

Table 3

Definition of the variables used for ANOVA

\begin{tabular}{|c|c|c|}
\hline An & \multicolumn{2}{|c|}{ Correspond à l'année de la campagne } \\
\hline Sais (saison) & \multicolumn{2}{|c|}{ Saison sèche : janvier à mai } \\
\hline & \multicolumn{2}{|c|}{ Saison humide : juin à décembre } \\
\hline Bathy (bathymétrie) & \multicolumn{2}{|c|}{$\left[0-5 \mathrm{~m}^{\circ}\right] ;[5-10 \mathrm{~m}] ;[10-15 \mathrm{~m}] ;[15-20 \mathrm{~m}] ;+20 \mathrm{~m}$} \\
\hline \multirow[t]{6}{*}{ Zone } & NW & Longitude $>14^{\circ} 30^{\prime} \mathrm{W}$ \\
\hline & & Latitude $>10^{\circ} 00^{\prime} \mathrm{N}$ \\
\hline & Centre & Longitude entre $13^{\circ} 30^{\prime} \mathrm{W}$ et $14^{\circ} 30^{\prime} \mathrm{W}$ \\
\hline & & Latitude entre $9^{\circ} 30^{\prime} \mathrm{N}$ et $10^{\circ} 30^{\prime} \mathrm{N}$ \\
\hline & $\mathrm{SE}$ & Longitude $<14^{\circ} 00^{\prime} \mathrm{W}$ \\
\hline & & Latitude $<9^{\circ} 30^{\prime} \mathrm{N}$ \\
\hline ITF (température de fond) & \multicolumn{2}{|c|}{ Par tranche de $10^{\circ} \mathrm{C}$} \\
\hline ISF (salinité de fond) & \multicolumn{2}{|c|}{ Par tranche de $1 \%$ o } \\
\hline
\end{tabular}

salinité de fond et la température de fond. Le modèle peut donc s'écrire de la manière suivante :

$$
I=\sum_{i} E_{i}+\varepsilon
$$

où $I$ représente l'indice de biodiversité considéré, $E_{i}$ l'effet statistique du facteur $i$ parmi les facteurs précédemment cités, $\varepsilon$ représente le terme résiduel.

La détection des effets significatifs s'effectue grâce à la procédure GLM de SAS (SAS Institute Inc.SAS/STAT ${ }^{\mathrm{TM}}$ ). Elle inclut, pour chaque modèle analysé, un double test de significativité des différents effets statistiques selon une procédure «Backward» et une procédure « Forward».

La procédure LSMEANS estime la valeur des différents effets statistiques du modèle par un ajustement selon la méthode des moindres carrés. L'analyse conduit ainsi à estimer les valeurs moyennes des différents indicateurs dans chacune des strates spatiales (classes de bathymétrie et de zone) et temporelles (année et saison hydrologique). En particulier, la répartition spatiale des indices est ainsi abordée de manière discrète.

\section{Résultats}

\subsection{Les modèles retenus}

En toute rigueur, les tests statistiques de l'analyse de variance sont valides et les estimations statistiques optimales sous hypothèse de normalité des résidus. Notons que cette hypothèse est vérifiée pour tous les modèles retenus en utilisant la droite des quantiles de la loi normale.

Par souci de clarté et de concision, nous ne présentons ici que les résultats de l'analyse temporelle. Nous ne décrivons ni ne discutons la distribution spatiale des indices de biodiversité.

Le modèle additif retenu dans le traitement des données de l'échantillon pour décrire tous les indicateurs comprend les effets des facteurs : an, bathymétrie ${ }^{1}$ (pour S), zone (pour R) et an*bathymétrie Tableau 4). Il explique entre 20 et $23 \%$ de la variabilité des données selon la variable étudiée ce qui est relativement satisfaisant compte tenu du nombre d'individus statistiques.

On remarque tout d'abord que, parmi les facteurs étudiés, les facteurs incluant la température de fond et la salinité de fond ne sont pas significatifs que ce soit en procédure «Backward » ou «Forward». Ces facteurs de l'environnement ne semblent pas influencer directement la valeur de la biodiversité que ce soit la richesse spécifique, la diversité de Shannon ou l'équitabilité. Les indicateurs ne sont pas non plus soumis à l'influence de la saison. Cette constatation, outre l'information intrinsèque qu'elle apporte, permet par la suite de comparer des résultats issus de campagnes de saisons sèche ou humide sans distinction. Les facteurs relatifs à la zone géographique (facteurs zone et an*zone) sont plus souvent négligeables et parfois non significatifs.

\section{2. Évolution temporelle des indices}

Il résulte de l'analyse temporelle 2 types de trajectoires Fig. 3.

Les indices de diversité et d'équitabilité présentent des valeurs absolues qui diffèrent selon le mode de calcul (par trait ou par campagne), mais qui suivent des évolutions parallèles. Globalement, l'évolution temporelle de ces indicateurs est marquée par une légère décroissance tendancielle. On met aussi en évidence un signal fort (une chute brusque) en 1989. Les valeurs de début et de fin de période gardent cependant des niveaux comparables.

Le nombre total d'espèces récoltées par campagne affiche une tendance plus nette à la baisse. Ainsi, la richesse spécifi-

\footnotetext{
${ }^{1}$ En toute rigueur, les résultats de la strate bathymétrique [0-5 $\left.\mathrm{m}\right]$ sont sujets à caution. En effet, le tirant d'eau du N/O A. Nizery ne lui permet pas d'explorer correctement cette strate. Cependant, les résultats qui lui sont relatifs ne sont pas aberrants et nous avons préféré les garder tout en émettant les réserves de circonstance.
} 
Tableau 4

Étude des différents facteurs testés dans les modèles linéaires : $\mathrm{R}^{2}$ relatif au modèle retenu (incluant les seuls effets significatifs) et probabilité associée à chacun des facteurs (procédure «forward»). Les variables sont définies dans le tableau 3. L'astérisque * indique des effets croisés, ns indique que le facteur n'a pas d'effet significatif

Table 4

Table of the factors used in the linear models. $\mathrm{R}$ of the selected model (significant effects only) and associated probability for each factor. Variables are defined in Table 3. * indicates cross effects. ns indicates non significant effects

\begin{tabular}{lllllllll}
\hline & $\mathrm{R}$ & $\mathrm{An}$ & Bathy & An*bathy & Zone & An*zone & ITF & ITS \\
\hline $\mathrm{S}$ & $19,6 \%$ & $10^{-4}$ & $10^{-4}$ & $1,4.10^{-3}$ & $\mathrm{~ns}$ & $\mathrm{~ns}$ & $\mathrm{~ns}$ & $\mathrm{~ns}$ \\
$\mathrm{H}^{\prime}$ & $23,5 \%$ & $10^{-4}$ & $\mathrm{NS}$ & $10^{-4}$ & $\mathrm{~ns}$ & $\mathrm{~ns}$ & $\mathrm{~ns}$ & $\mathrm{~ns}$ \\
$\mathrm{R}$ & $20,0 \%$ & $10^{-4}$ & $\mathrm{NS}$ & $1,5.10^{-4}$ & $1,5.10^{-3}$ & $\mathrm{~ns}$ & $\mathrm{~ns}$ & $\mathrm{~ns}$ \\
\hline
\end{tabular}

que a clairement diminué dans l'échantillon entre 1985-1986 (environ 125 espèces différentes récoltées) et 1991-1992 (environ 80 espèces). La décroissance est statistiquement significative lorsque l'on s'intéresse au nombre d'espèces par trait. On peut en effet ajuster une droite de régression de pente négative. Le coefficient d'ajustement $R^{2}$ vaut 0,74 et il est significatif à $95 \%$ de certitude. La pente est significativement différente de $0(p<0,05)$.

Enfin, les diagrammes rang/fréquence montrent que les campagnes Chagui 8 et 9 de 1989 se démarquent du reste des campagnes dont les diagrammes ont des allures similaires Fig. 4.

\section{Discussion}

\subsection{Le système en début de période}

Avant de discuter des propriétés de stabilité du système (ici, en regard de sa biodiversité), il est nécessaire de caractériser la situation écologique (Grimm et Wissel, 1997). Le niveau de description de cette étude est celui du peuplement ichtyologique côtier. L'échelle est relativement large. Au niveau spatio-temporel, cela se traduit par la prise en compte des variations interannuelles de la biodiversité concernant l'ensemble de la zone côtière du littoral guinéen. À cette a)
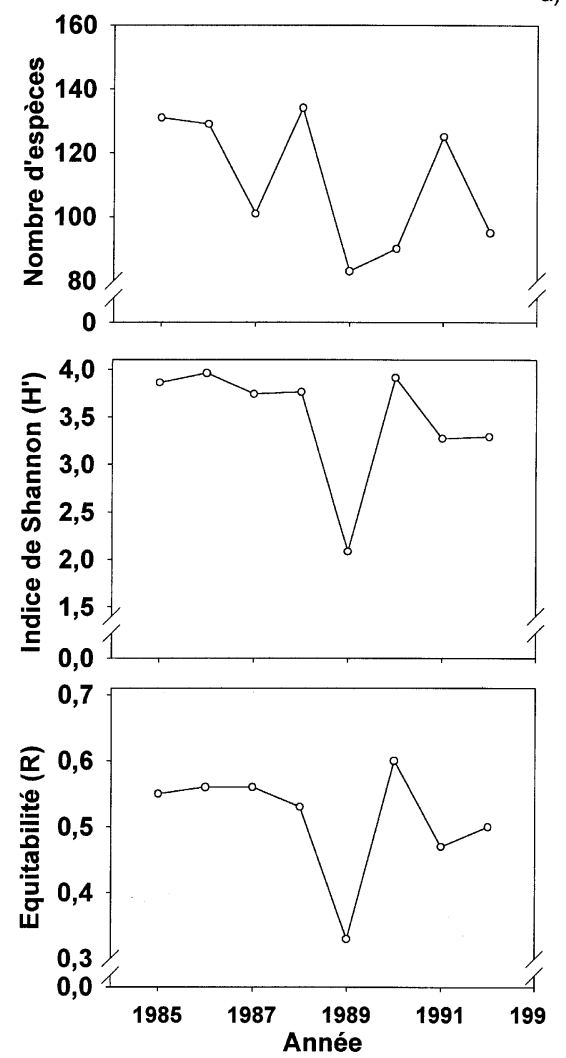

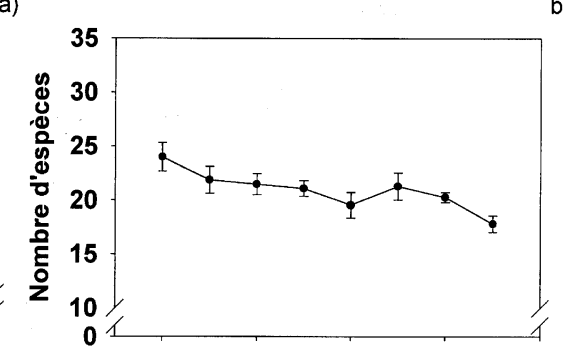

b)
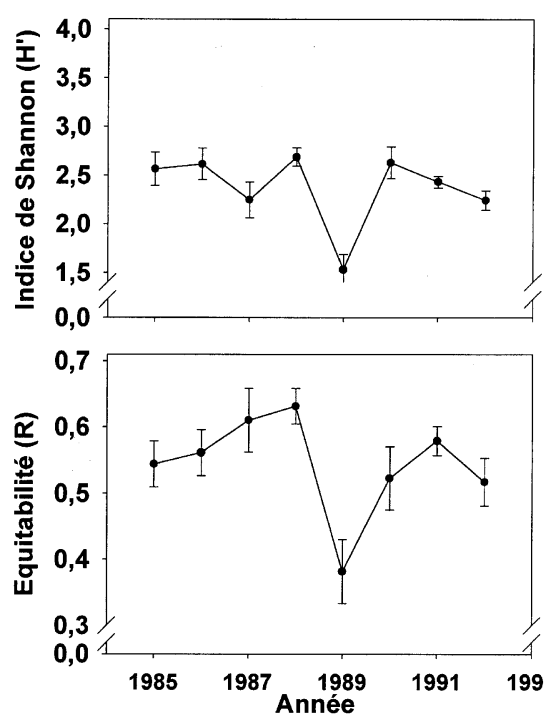
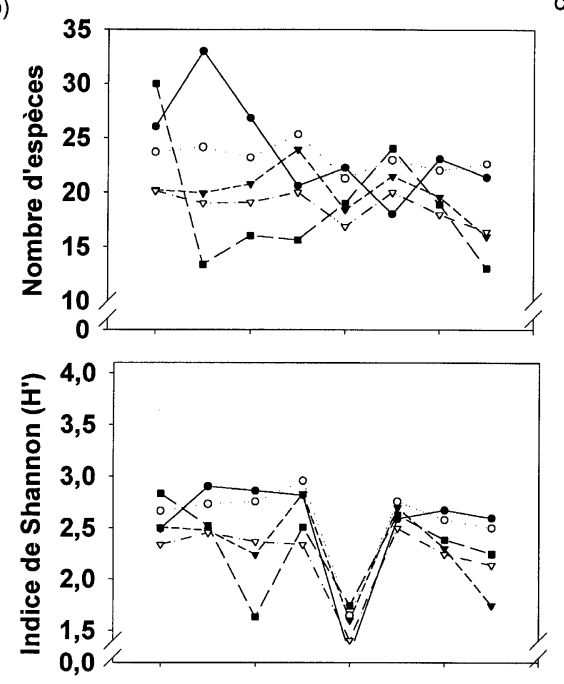

c)

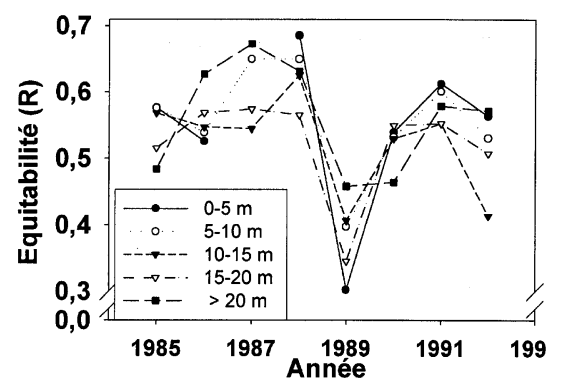

Fig. 3. Variations des indices de biodiversité en fonction du temps : trajectoires de la richesse spécifique, de la diversité de Shannon et de l'équitabilité. (a) indices calculés par campagne, (b) indices moyens par trait et (c) indices moyens par trait ventilés par strate bathymétrique.

Fig. 3. Variation of the biodiversity indexes during time: trajectories of species richness, Shannon and equitability indexes. (a) indexes by campaign, (b) mean indexes by trawl and (c) by trawl and depth stratum. 


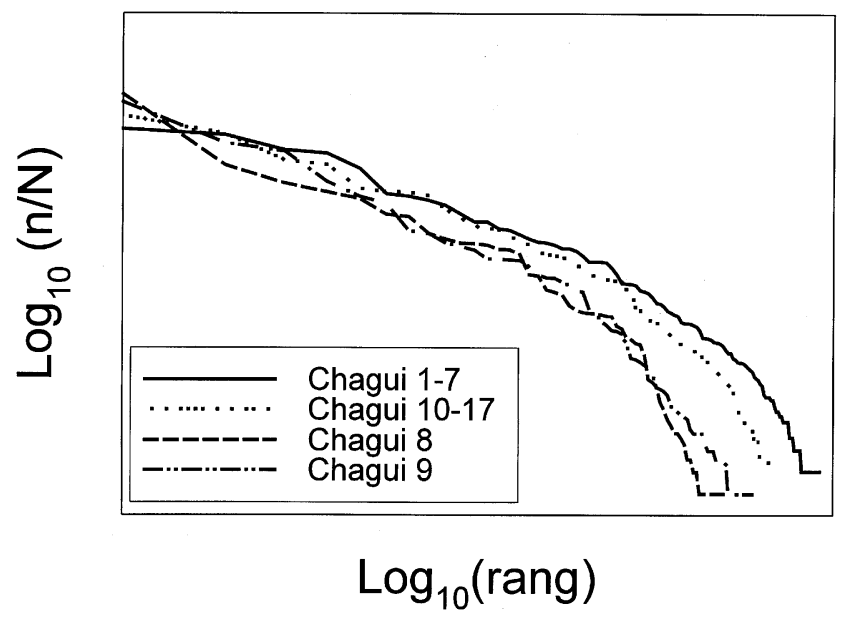

Fig. 4. Diagrammes rang/fréquence.

Fig. 4. Rank/frequencies diagrams.

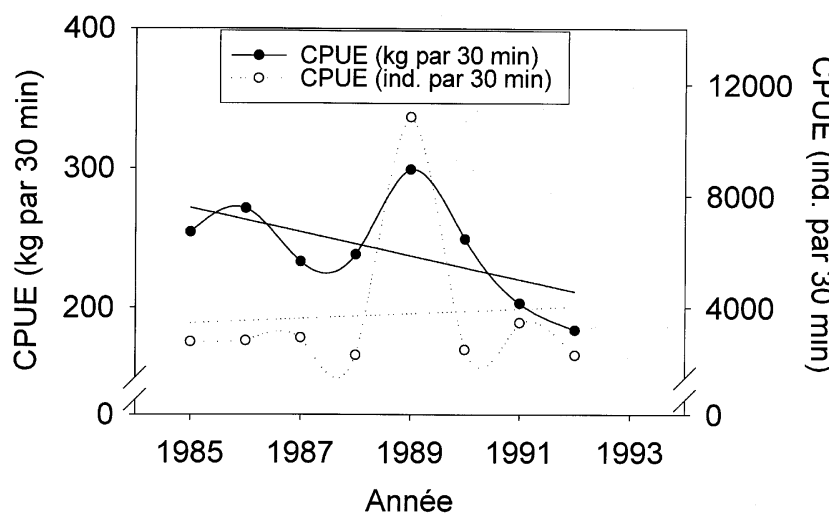

Fig. 5. Évolutions des CPUE exprimées en kg et en nombre d'individus par trait. Les tendances apparaissent pour chacune des 2 courbes.

Fig. 5. Evolution of CPUE in $\mathrm{kg}$ and in number of individuals per trawl. The trends are plotted for the 2 curves.

échelle et compte tenu du développement extrêmement rapide de l'exploitation des ressources halieutiques en Guinée, il paraît pertinent de situer l'état de référence aux années 1985-1986.

Il n'existe pas de seuils utilisables pour établir de diagnostic à partir de la valeur des différents indices mais, si l'on se réfère à divers exemples cités notamment par Frontier (1983) et Barbault (1992), le système apparaît diversifié en début de période. Au niveau du calcul par campagne, la valeur de $\mathrm{H}^{\prime}$ vaut 3,9 et celle de $\mathrm{R}$ est de 0,55. Au niveau moyen par trait, les valeurs sont plus modestes mais restent cependant raisonnables en liaison avec le caractère limité de la surface chalutée à chaque trait. Cet aspect est confirmé par l'allure des diagrammes rang/fréquence qui montrent une diversité et une régularité élevées. Ces diverses indications sont caractéristiques d'un système mature en début de période.

\subsection{L'évolution temporelle des indices}

Les perturbations subies par le système sont essentiellement dues à l'accroissement de l'effort de pêche. Pour Gascuel et al. (1997), les stocks halieutiques et en particulier les
Tableau 5

Liste des principales espèces non observées en 1991-1992 par ordre d'importance en 1985-1986

Table 5

List of the main non observed species in 1991-1992

\begin{tabular}{|c|c|c|}
\hline \\
\hline \multicolumn{3}{|l|}{$\frac{\text { Espèces }}{\text { Sepiia } \mathrm{sp} .}$} \\
\hline Engraulis encrasicolus & Sphyranea sphyranea & Uranoscopus polli \\
\hline Stephanolepis hispidus & $\begin{array}{l}\text { Chilomycterus } \\
\text { spinosus }\end{array}$ & $\begin{array}{l}\text { Epinephelus } \\
\text { alexandrinus }\end{array}$ \\
\hline Dentex gibbosus & Saurida brasiliensis & Scomber japonicus \\
\hline Sphoeroïdes spengleri & Diodon hystrix & Rypticus saponaceus \\
\hline Sphyranea sp. & Scarus hoefleri & Diodon holocanthus \\
\hline Bothus podas & Trachurus trechae & Fistularia petimba \\
\hline Serranus accraensis & Lutjanus fulgens & Epinephelus goreensis \\
\hline
\end{tabular}

populations de la communauté à Sciaenidés sont dans une situation qualifiée de surexploitation globale. Cette situation se traduit par une diminution des captures. L'échantillonnage met en avant une diminution des CPUE en terme de biomasse (confirmée par plusieurs auteurs dont Gascuel et al., 1997 ou Domain et al., 2000) tandis que le nombre d'individus capturés reste sensiblement constant sur la période (Fig. 5). Le système ne peut donc être qualifié de résistant car les biomasses ne sont pas conservées (McGillivray et Grime, 1995).

Cette chute des captures s'accompagne d'une baisse assez nette de la richesse spécifique. Plusieurs espèces, rares en début de période, disparaissent de l'échantillonnage en 1991-1992 Fig. 6). Ce sont essentiellement des espèces issues de la communauté à Sparidés et des autres communautés du large évoquées par Domain (1989); mais elles étaient présentes dans les zones les plus littorales en 1985-1986 car elles sont recensées dans les échantillonnages de début de période Fig. 77. Si l'effet statistique de l'échantillonnage peut être discuté, il semble donc peu probable qu'il ne s'agit là que d'un artefact dû au biais évoqué plus haut. En outre, une correction a déjà été effectuée sur la zone prise en

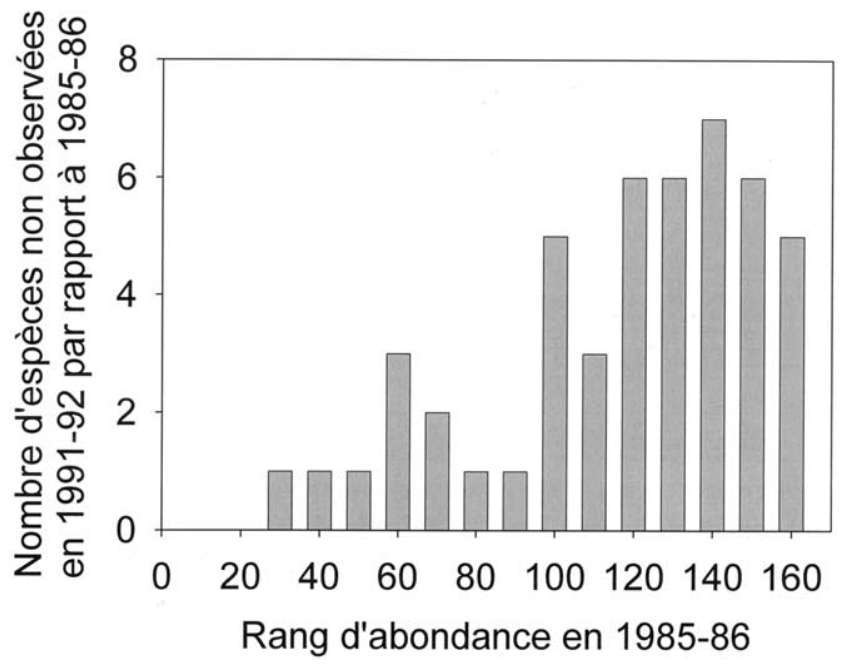

Fig. 6. Rang d'abondance en 1985-1986 des espèces qui n'ont pas été observées en 1991-1992.

Fig. 6. Class of abundance in 1985-1986 of the species that have not been observed in 1991-1992. 


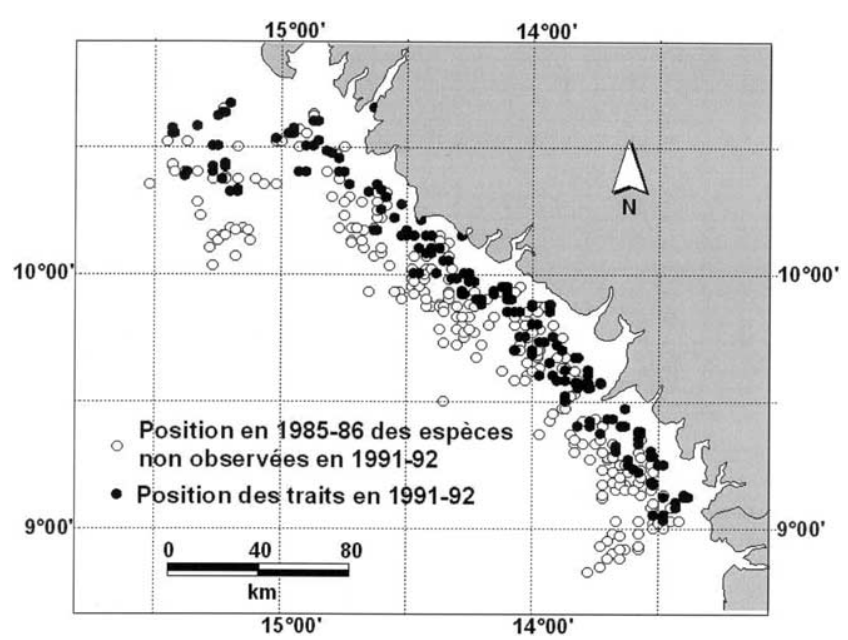

Fig. 7. Répartition géographique en 1985-1986 du groupe d'espèces qui ont disparu de l'échantillonnage en 1992.

Fig. 7. Spatial distribution in 1985 of the species that were not observed in 1992.

compte. Cette correction a d'ailleurs eu comme conséquence une augmentation artificielle, a posteriori, de l'effort d'échantillonnage sur la partie la plus côtière entre 1985-1986 et 1991-1992 (186 traits contre 508). Cela accentue le problème posé par la non détection de ces espèces.

Il est donc probable que les biomasses résiduelles de certaines espèces soient passées sous un «seuil de détection » dans la zone échantillonnée Fig. 8. Ceci pourrait être directement lié à la pression halieutique exercée sur l'ensemble des ressources et à la décroissance générale des biomasses qui en découle. Et ce, même si les espèces en question Tableau 5) ne sont, pour la plupart, pas directement visées par l'exploitation du plateau (moins d'une dizaine sur 48 a une importance économique certaine). L'impact de la pêche est donc largement ressenti par l'ensemble du peuplement. On peut aussi imaginer que la pression halieutique a modifié leur zone de répartition en provoquant une rétraction de celle-ci vers le large. La pression de pêche et une certaine non-sélectivité des pratiques de pêche peut, en effet, conduire à une nouvelle compétition entre les espèces pour

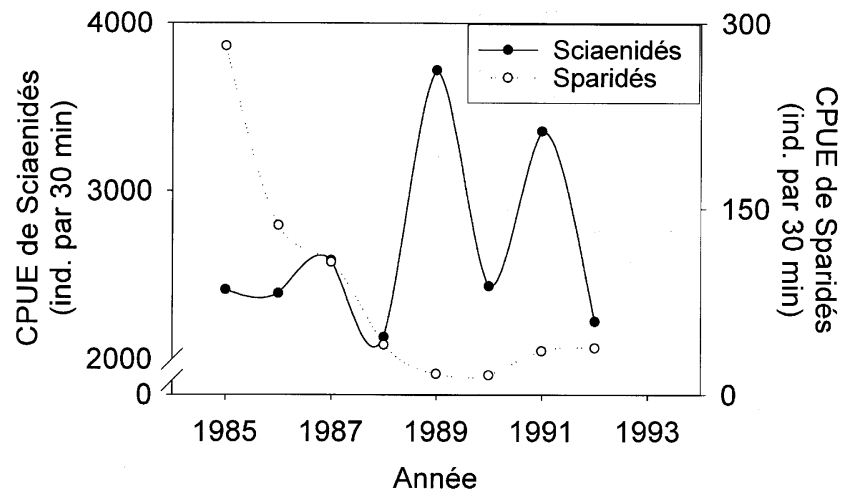

Fig. 8. Évolution de l'abondance des communautés ichtyologiques à Sciaenidés et Sparidés.

Fig. 8. Evolution of the abundance of the Sciaenid and Sparid fish communities. l'espace et la ressource et induire de profonds changements dans les communautés (Jennings et Polunin, 1996) et leurs structures. Il n'est pas exclu que ces modifications soient dues à des changements environnementaux mais Baran (1995) ou Domain (2000) ne mentionnent rien de particulier en ce sens.

Dans le même temps, si l'ensemble des indicateurs de biodiversité montre une décroissance tendancielle, cette décroissance apparaît nettement plus marquée si l'on considère la richesse spécifique que si l'on considère la diversité et l'équitabilité. En effet, malgré cette tendance et un signal fort en 1989, les valeurs de début et de fin de période de H' et $\mathrm{R}$ gardent des niveaux comparables (calcul par campagne et par trait). En outre, après l'épisode de 1989 sur lequel nous reviendrons, ils retrouvent des valeurs assez proches de leurs valeurs initiales. Ceci pourrait être interprété comme le signe d'une certaine résilience du système. Ainsi, les ressources démersales du plateau continental guinéen semblent former un système mature et résilient en regard de sa biodiversité. Cette relative stabilité trouve son explication à plusieurs niveaux. Tout d'abord, comme le signale Ricotta (2000), de par son calcul, l'indice de Shannon est assez insensible à la présence (ou l'absence) d'espèces rares et donc ici à la disparition de l'échantillonnage d'espèces déjà peu abondantes à l'origine. Il n'en reste pas moins que certaines espèces moins rares ont aussi disparu. Cette disparition du nombre d'espèces présentes ne semble pas s'accompagner d'une diminution du nombre d'individus. Il est donc probable que cette diminution de la richesse spécifique a été « compensée » par une augmentation des effectifs et que cette augmentation des effectifs n'est pas le fait uniquement des espèces les plus abondantes ce qui aurait sans doute entraîné une chute plus nette de la diversité et de l'équitabilité comme lors de l'épisode de 1989.

En 1989, on note une brusque chute de tous les indicateurs (même la richesse spécifique atteint sa valeur la plus faible sur l'ensemble de la période). L'explication la plus fréquemment avancée est liée à des raisons environnementales. En effet, des pluies exceptionnelles fin 1988, début 1989 auraient entraîné un apport terrigène particulièrement important de la part des grands fleuves guinéens. Ces apports auraient favorisé le recrutement de certaines espèces de la communauté à Sciaenidés d'estuaires tandis que celui d'autres espèces d'affinité estuarienne moindre aurait été déplorable en raison d'un effet dit « de chasse » (Domain, 2000). Domain compare l'impact de ces apports terrigènes à l'effet d'un upwelling, en terme de fenêtre environnementale optimale. De fait, les fréquences relatives des différentes espèces présentes ont été profondément modifiées : quelques espèces ont connu un recrutement exceptionnellement élevé ; d'autres ont vu leurs effectifs chuter. Aussi, l'explosion des effectifs et des biomasses n'est due qu'à quelques espèces. Ceci affecte bien évidemment la diversité du système et la valeur de H'. Cette nette dominance d'une poignée d'espèces est particulièrement bien traduite par le diagramme rang/fréquence lors de la campagne Chagui 08. Il convient 
toutefois de rester prudent sur la capacité de réaction du système à un événement hydroclimatique exceptionnel en raison du caractère ponctuel, sur une seule campagne, de l'observation.

\section{Conclusion}

Étudier la biodiversité à travers des indices synthétiques, comme nous l'avons fait, revient à étudier un écosystème au travers d'indicateurs émergents de l'état de diversité de sa biocénose. Si l'on admet l'intérêt de ceux-ci pour diagnostiquer «l'état de santé écologique » du système, notre étude met ainsi en évidence une situation apparemment paradoxale en Guinée. D'un côté, les ressources démersales du plateau continental semblent former un système globalement résilient sur la période, sous une pression de pêche croissante. De l'autre, l'impact de la pêche sur l'abondance (en biomasse) et sur la richesse spécifique est assez conséquent mais il semble compensé par une augmentation des effectifs pour la plupart des espèces. On pourrait être tenté d'avancer que la structure des communautés ichtyologiques concernées et les relations fonctionnelles sont préservées ou que le jeu des espèces redondantes permet au système de garder un certain niveau d'entropie. Plus sûrement, il convient d'être prudent sur l'approche que nous avons utilisée. En particulier, la pertinence de tels indices (et notamment l'indice de Shannon) pour quantifier la biodiversité est souvent remise en cause et les suggestions pour améliorer la qualité des indices abondent dans la littérature (voir Heip et al., 1998 ; Izsak et Papp, 2000 ; Ricotta, 2002). Une telle étude, si elle ne semble donc pas suffisante pour évaluer «l'état écologique »du système peut néanmoins s'avérer intéressante dans le cadre d'une approche de précaution (Gascuel et Ménard, 1997). Elle permet d'aborder l'aspect systémique de la gestion des ressources marines exploitées car, comme le souligne Frontier (1999), « la diversité taxonomique ne reflète pas exactement la diversité fonctionnelle mais elle reste quand même, d'un point de vue holiste, un indicateur de la complexité du système ». En particulier dans le cas des ressources du plateau continental guinéen, elle permet d'avoir une vue d'ensemble du peuplement et des conséquences de l'augmentation significative de la pression de pêche sur le peuplement et de ne pas uniquement se focaliser sur quelques populations d'espèces d'intérêt commercial.

\section{Remerciements}

Nos remerciements sont adressés à l'IRD et au CNSHB pour avoir fourni les données nécessaires à la réalisation de ce travail ainsi qu'à l'ensemble du département halieutique de l'ENSAR. Merci à Eric Rochard et Mario Lepage pour leur contribution. Nous tenons à remercier aussi le relecteur anonyme pour ses remarques qui ont contribué à améliorer la qualité du manuscrit.

\section{Références}

Baran, E., 1995. Dynamique spatio-temporelle des peuplements de poissons estuariens en Guinée : Relations avec le milieu abiotique. Thèse, Université de Bretagne Occidentale. TDM 142, ORSTOM, Paris.

Barbault, R., 1992. Écologie des peuplements, structure, dynamique et évolution. Masson, Paris.

Blanchard, F., 2001. Une approche de la dynamique des peuplements de poissons démersaux exploités : analyse comparée de la diversité spécifique dans le golfe de Gascogne (océan Atlantique) et dans le golfe du Lion (mer Méditerranée). Aquat. Living Resour. 14, 29-40.

Chavance, P., Diallo, A., Diallo, M., Domain, F., Drapeau, L., Fautrel, V., Guilavogui, A., Morize, E., Solie, K., Tarore, S., 1997. Atlas des pêches maritimes de Guinée 1997. Institut Français de Recherche Scientifique pour le Développement en Coopération (ORSTOM) Conakry, Centre National des Sciences Halieutiques de Boussoura (CNSHB), Paris.

Domain, F., 1989. Rapport des campagnes de chalutages du N.O. A. Nizery dans les eaux de la Guinée de 1985 à 1988. Doc. Sci. Centre recherche halieutique, Boussoura (Guinée).

Domain, F., 2000. Influence de la pêche et de l'hydroclimat sur l'évolution dans le temps du stock côtier (1985-1995). In: Domain, F., Chavance, P., Diallo, A. (Eds.), La pêche côtière en Guinée : ressources et exploitation. CNSHB IRD, Boussoura (Guinée) Paris (France).

Domain, F., Chavance, P., Diallo, A. (Eds.), 2000. La pêche côtière en Guinée : ressources et exploitation. CNSHB IRD, Boussoura (Guinée) Paris (France).

Fager, W., Longhurst, A.R., 1968. Recurrent Group Analyses of species assemblages of demersal fishes in the gulf of Guinea. L. Fish. Res. Board Can. 25, 1405-1421.

Frontier, S., coord, 1983. Stratégies d'échantillonnage en écologie. Masson / Les presses de l'Université Laval, Québec.

Frontier, S., 1999. Les Écosystèmes. Presse Universitaires de France, Coll. Que sais-je ?, Paris.

Gascuel, D., Domain, F., Chavance, P., 1997. L'exploitation de la communauté à Sciaenidés de Guinée : un premier diagnostic sur l'état des stocks, par modélisation linéaire de l'abondance (1985-92). Troisième forum halieumétrique, Montpellier 1-3 juillet 1997.

Gascuel, D., Ménard, F., 1997. Assessment of a multispecies fishery in Senegal, using production models and diversity indices. Aquat. Living Resour. 10, 281-288.

Grimm, V., Wissel, C., 1997. Babel, or the ecological stability discussions: An inventory and analysis of terminology and a guide for avoiding confusion. Oecologia 109, 323-334.

Heip, C.H.R., Herman, P.M.J., Soetaert, K., 1998. Indices of diversity and eveness. Océanis 24, 61-87.

Izsak, J., Papp, L., 2000. A link between ecological diversity indices and measures of biodiversity. Ecol. Modelling 130, 151-156.

Jennings, S., Polunin, N.V.C., 1996. Impacts of fishing on tropical reefs ecosystems. Ambio 25, 44-49.

Jouffre, D., Domain, F., 2000. Les ressources démersales de la zone côtière du plateau continental guinéen : éléments de la structure temporelle du peuplement ichtyologique. In: Domain, F., Chavance, P., Diallo, A. (Eds.), La pêche côtière en Guinée, Ressources et exploitation. CNSHB, IRD, Boussoura (GIN), Paris (FRA).

Koslow, J.A., Hanley, F., Wicklend, R., 1988. Effect of fishing on reef communities at Pedro Bank and Port Royal Cays, Jamaica. Mar. Ecol. Prog. Ser. 43, 201-212.

Lévêque, C., 1994. Le concept de biodiversité : de nouveaux regards sur la nature. Nat. Sci. Soc. 2, 243-254.

Margalef, R., 1958. Information theory in ecology. Gen. Syst. 3, 3671.

May, R.M., 1976. Patterns in multispecies communities. In: May, R.M. (Ed.), Theoretical Ecology. W.B. Saunders Co, Philadelphia, pp. $142-162$.

McGillivray, C.W., Grime, J.P., 1995. Testing predictions of the resistance and resilience of vegetation subjected to extreme events. Funct. Ecol. 9, 640-649. 
Naeem, S., Li, S., 1997. Biodiversity enhances ecosystem reliability. Nature $390,507-509$.

Pielou, E.C., 1966. The measurement of diversity in different types of biological collections. J. Theor. Biol. 13, 131-144.

Ricotta, C., 2000. From theoretical ecology to statistical physics and back: self-similar landscape metrics as a synthesis of ecological diversity and geometrical complexity. Ecol. Model. 125, 245-253.
Ricotta, C., 2002. Bridging the gap between ecological diversity indices and measures of biodiversity with Shannon's entropy: comment to Izsak and Papp. Ecol. Model. 152, 1-3.

Russ, G.R., Alcala, A.C., 1998. Natural fishing experiments in Marine Reserves 1983-1993: communities and trophic response. Coral Reefs 17, 383-397.

Shannon, C.E., 1948. A mathematical theory for communication. Bell Syst. Tech. J. 27 379-423 et 623-656. 